\title{
Influence of Specimen Geometry on the Estimation of the Planar Biaxial Mechanical Properties of Cruciform Specimens
}

\author{
J.-J. Hu • G.-W. Chen • Y.-C. Liu • S.-S. Hsu
}

Received: 5 June 2013 / Accepted: 7 November 2013 / Published online: 14 December 2013

(C) Society for Experimental Mechanics 2013

\begin{abstract}
It is in general challenging to characterize planar mechanical properties of extremely soft tissues such as cellseeded collagen gels. One of the difficulties is related to premature failure of specimens. This issue may be resolved by employing fillets on stress-concentrated spots of the specimen. The existence of fillets, however, complicates the estimation of stress at the center of the specimen where stiffness data are collected. In this study, cruciform rubber specimens with two types of fillets (general vs. cut-in fillets) at the intersections of perpendicular arms were prepared and subjected to planar biaxial mechanical testing, aiming at investigating how the fillets affect the estimation of mechanical properties of cruciform specimens. Digital image correlation was used to analyze full-field deformation in the central region of the specimens. Finite element analysis with a Neo-Hookean model was performed to simulate the full-field deformation under the same experimental boundary conditions. The strain distribution for each specimen geometry obtained by finite element analysis was found to be in good agreement with that analyzed by digital image correlation, validating the finite element models. Finite element simulation showed that the greatest value of the maximum principal strain decreased with
\end{abstract}

Electronic supplementary material The online version of this article (doi:10.1007/s11340-013-9826-2) contains supplementary material, which is available to authorized users.

J.-J. $\mathrm{Hu}(\bowtie) \cdot$ G.-W. Chen · S.-S. Hsu

Department of Biomedical Engineering, National Cheng Kung

University, \#1 University Rd., Tainan, Taiwan 701

e-mail: jjhu@mail.ncku.edu.tw

J.-J. Hu

Medical Device Innovation Center, National Cheng Kung

University, Tainan, Taiwan

Y.-C. Liu

Department of Mechanical Engineering, National Chiao Tung

University, Hsinchu, Taiwan increasing the fillet radius regardless of the fillet type. Increasing the fillet radius, in general, also reduced the strain field uniformity in the central region. Compared with general fillets, however, the use of cut-in fillets provided greater strain field uniformity given the same fillet radius. Finite element analysis was also used to estimate effective transverse length required to convert tensile force at the boundary to local stress at the center. It was found that the effective transverse length for each specimen geometry remained relatively constant if the specimen was not excessively deformed (i.e., global equibiaxial stretch $\leq 1.2$ ). We suggest using cut-in fillets at the intersections of perpendicular arms when preparing cruciform specimens for testing extremely soft tissues.

Keywords Planar biaxial mechanical testing · Specimen geometry $\cdot$ Fillets $\cdot$ Digital image correlation $\cdot$ Finite element method $\cdot$ Soft tissues

\section{Introduction}

Cell-seeded collagen gels have been used as a model system to study cell-matrix interactions in tissue morphogenesis and wound healing [1-3]. In tissue engineering, the property of directed contraction in uniaxially constrained, rectangular, cell-seeded collagen gels has been used to engineer highly anisotropic load-bearing tissues [4-6]. The mechanical properties of collagen gels in these studies were measured by unaxial tensile testing, which, however, is insufficient to fully characterize the mechanical behavior of the gel if the gel is mechanically anisotropic. Recently, the model was further used to study tissue development under various planar biaxial mechanical constraints and planar biaxial mechanical properties of the gels were determined in these studies [7, 8].

It is in general challenging to characterize planar mechanical properties of extremely soft tissues such as cell-seeded 
collagen gels. These tissues usually have limited suture retention strength so that it is not practical to stretch specimens via arrays of sutures; this method has been widely used for testing soft tissues since its first use by Lanir and Fung [9]. Others prepared cruciform specimens and used end-clamps to couple the specimen to the loading system $[8,10]$. For extremely soft tissues, one of the difficulties related to this method is the premature failure of specimens. This issue may be resolved by employing fillets on stress-concentrated spots of the specimen. The existence of fillets, however, makes the crosssectional area of the central region ill-defined. Also, the nonuniformity of stress/strain field may further complicate the estimation of stress at the center of the specimen where stiffness data are collected.

For standard uniaxial tensile testing, the conversion of tensile force to stress is straightforward as in the central region of a dumbbell-shaped specimen stress/strain field is uniform and cross-sectional area is well-defined. Stiffness data can thus be easily obtained. The geometry of cruciform specimens used for planar biaxial mechanical testing, however, has not been standardized. It has been shown that boundary effects have significant influence on the estimation of the planar biaxial mechanical properties of soft tissues [11, 12]. Little has been done to improve cruciform specimen design with respect to the reliability of stiffness data in the field of soft tissue testing. Particularly for extremely soft tissues, it is required to seek a compromise that the premature failure is prevented and measured stress and strain reasonably reflects the real mechanical behavior of the specimen.

In this study, we used rubber as a model material and prepared cruciform specimens with two types of fillets (general vs. cut-in fillets) at the intersections of perpendicular arms for investigating how the fillets affect the estimation of mechanical properties of cruciform specimens. Digital image correlation was used to analyze full-field deformation in the central region of the specimens. On the other hand, finite element analysis with a Neo-Hookean model was performed to simulate the full-field deformation under the same experimental boundary conditions; the only material parameter of the Neo-Hookean model was obtained by a simple inverse procedure prior to the analysis. Finite element analysis was also used to estimate the effective transverse length required to convert prescribed force at the boundary to local stress at the center.

\section{Methods}

Mechanical Testing of Cruciform-Shaped Rubber Specimens

A modified version of a planar biaxial mechanical tester [13] was used. Specifically, a high-resolution CCD $(2448 \times 2050$; GC2450, Prosilica, Germany) with a lens (HF25SA-1, Fujinon) was used for tracking full-field deformation and a pair of commercial load cells (WMCP-1000G, Interface, Scottsdale, AZ) were used for measuring tensile forces in perpendicular stretching directions. Cruciform specimens with two fillet types (general fillets: R0625, R1250, R2500, cut-in fillets: In_R03125, and In_R0625; see Fig. 1) at the intersections of perpendicular arms were cut from thin, black rubber sheets $(0.3 \mathrm{~mm}$ thick) and subjected to mechanical testing. Briefly, high-contrast speckles for tracking surface displacement were created by spraying white paint on the specimen. The specimen was clamped at the ends of its four arms to the loading assemblies of the tester. When the specimen was unloaded, an image of the central region was taken as the reference image. A displacement-controlled testing protocol, in which global stretches in the stretching directions were kept the same, was then performed. Deformed images of the central region were taken periodically and corresponding tensile forces in the stretching directions were recorded. The fullfield deformation of the central region was analyzed by processing the images as follows.

\section{Digital Image Correlation and Strain Field Estimation}

A custom code in LabVIEW was written to measure the fullfield deformation; coding in LabVIEW allows easy integration of strain field estimation with mechanical testing. The code was initiated by opening the reference image, on which a rectangular (nearly square) region of interest (ROI) was manually selected. Nodes were created within the ROI (red dots in Fig. 2(a)) and node-centered patterns (size varying from $24 \times$ 24 pixels to $34 \times 34$ pixels for different specimens) were saved in memory for tracking the motion of nodes. The code then began to analyze the first deformed image. Digital image correlation (DIC) was performed by using pattern match 2.vi (a NI Vision function), which searched a nearby area of the original position of a node in the image for the best fit of its pattern. The function allows subpixel registration up to $1 / 25$ pixel as well as in-plane rotation less than $4^{\circ}$. If a good fit was found, then it became the new position of the node. The searching process continued until new positions of all the nodes were determined. For strain field estimation of the deformed image, the (initial) positions of the nodes in the reference image were used to mesh the ROI into triangular elements by DelaunayTri.m (an embedded MATLAB function) (Fig. 2(b)). Given the respective positions of the three vertices (nodes) of a triangular element in the reference image and in the deformed image, the deformation gradient of the element was determined (see Appendix A). Once deformation gradients of all the elements were obtained, Green strain $\left(\mathrm{E}_{11}\right.$, $\mathrm{E}_{22}, \mathrm{E}_{12}$ ), rigid-body rotation angle $(\phi)$ [14], and the maximum and minimum principal strains $\left(\mathrm{E}_{\mathrm{p}, \max }, \mathrm{E}_{\mathrm{p}, \mathrm{min}}\right)$ of all the element were calculated, generating full-field strain distributions. Herein, $\mathrm{E}_{\mathrm{p}, \max }$ was used as a failure index while the ratio of the principal strains $\left(\mathrm{E}_{\mathrm{p}, \max } / \mathrm{E}_{\mathrm{p}, \min }\right)$ was used as an 


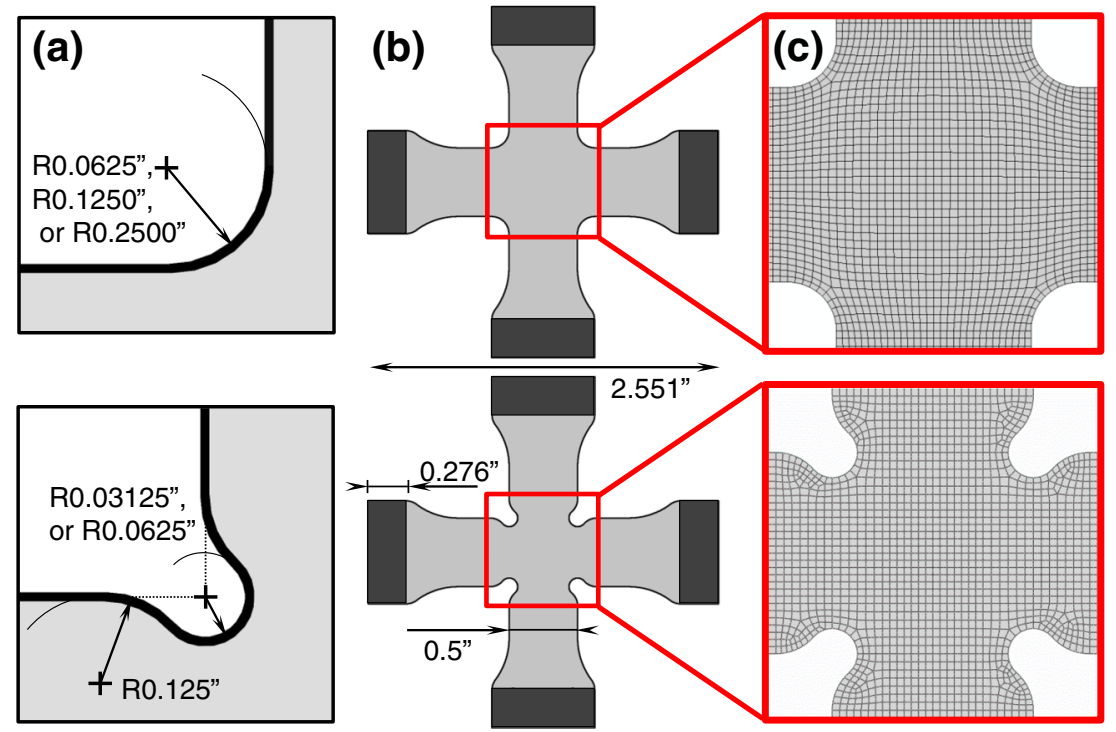

Fig. 1 Schematic diagram showing the dimensions of cruciform specimens with the general and the cut-in fillets at the intersections of perpendicular arms (top and bottom panels, respectively) (a, b) and the meshes in the central region for finite element analysis (c). The center of curvature for the general fillet is located away from the intersection of edges depending on the radius; three radii of curvature were tested in this group (R2500: R0.2500", R1250: R0.1250", and R0625: R0.0625") while that for the cut-in fillet is right at the intersection; two radii of curvature were tested in this group (In_R03125: R0.03125" and In_R0625: R0.0625"). The dark shadow areas indicate the clamped portion of the specimen. Note that the meshes shown here were created with approximate global size of $5 \mathrm{~mm}$ for illustration

index for strain field uniformity. The code then analyzed the next deformed image by the same algorithm with the new positions of the nodes. The whole process repeated until the full-field strain distributions of the last deformed image were determined (Fig. 2(c)).

\section{Constitutive Modeling of the Rubber Specimens}

Partly because of the existence of fillets and hence the illdefined cross-sectional area in the central region, it is difficult to estimate stress at the center of the specimen where stiffness data are collected. The tensile forces in the stretching directions $\left(f_{1}\right.$ and $\left.f_{2}\right)$ and the associated normal Green strains at the center of the specimen $\left(E_{11}^{\exp }\right.$ and $\left.E_{22}^{\exp }\right)$ were thus used to establish the constitutive relation with the help of finite element analysis.

Finite element models were created and analyzed using ABAQUS (Pawtucket, RI). The rubber was modeled as an incompressible isotropic hyperelastic material using the NeoHookean strain energy function, which is defined as:

$W\left(\lambda_{1}, \lambda_{2}, \lambda_{3}\right)=\mathrm{C}_{10}\left(\lambda_{1}^{2}+\lambda_{2}^{2}+\lambda_{3}^{2}-3\right)$

where $\mathrm{C}_{10}$ is a material parameter and $\lambda_{i}$ are the principal stretches. The specimen, particularly around the fillets, was first partitioned into shapes that are less complex for better meshing results. Because the specimen is thin with negligible bending stiffness, CPS8R, the 8-node quadratic plane stress element with reduced integration, were used to mesh the geometry. Based on a mesh convergence study (see Supplemental Figure 1), the approximate global size was set to be $0.3 \mathrm{~mm}$ and typically a total of $\sim 20,000$ elements were generated for each specimen geometry. Note that the clamped portion of each arm was treated as rigid body. The tensile forces in the stretching directions, which were simplified as concentrated forces acting on the clamped portion of each arm, were assigned as the boundary conditions. The large deformation was taken into account in the simulation by using the NLGEOM option. The full-field deformation was then analyzed. As our desired strain measure is Green strain (the default strain output of ABAQUS is the true strain, $\varepsilon=\ln \lambda$ ), we extracted deformation gradient by calling GETVRM in UVARM (an ABAQUS user subroutine). The Green strain was then calculated.

As we had experimental data of $f_{1}$ and $f_{2}$ and associated $E_{11}^{\exp }$ and $E_{22}^{\exp }$ in a number of equilibrium configurations, we simulated the normal Green strains at the center of the specimen $\left(\mathrm{E}_{11}^{\text {fem }}\right.$ and $\mathrm{E}_{22}^{\text {fem }}$ ) for each set of $f_{1}$ and $f_{2}$ with a guess value of $\mathrm{C}_{10}$ and determined manually the best-fit $\mathrm{C}_{10}$ that gives the minimum of the objective function: $e=\sum_{k=1}^{m}\left\{\left(\mathrm{E}_{11}^{\exp }-\mathrm{E}_{11}^{\text {fem }}\right)_{k}^{2}+\left(\mathrm{E}_{22}^{\text {exp }}-\mathrm{E}_{22}^{\text {fem }}\right)_{k}^{2}\right\}$ where $m$ is the number of equilibrium configurations. Note that this approach can be performed manually because only one material parameter is involved in the model and only interpolated strains at the center of the specimen were considered in the objective function. 
Fig. 2 The procedure of digital image correlation. (a) After manually selected a rectangular ROI, nodes (dots in red) are virtually created over the ROI by a simple algorithm. (b) Triangular elements, on which the deformation gradients are determined, are established based on positions of the nodes using DelaunayTri.m. (c) Digital image correlation was performed on deformed images taken at a series of equilibrium states (a)
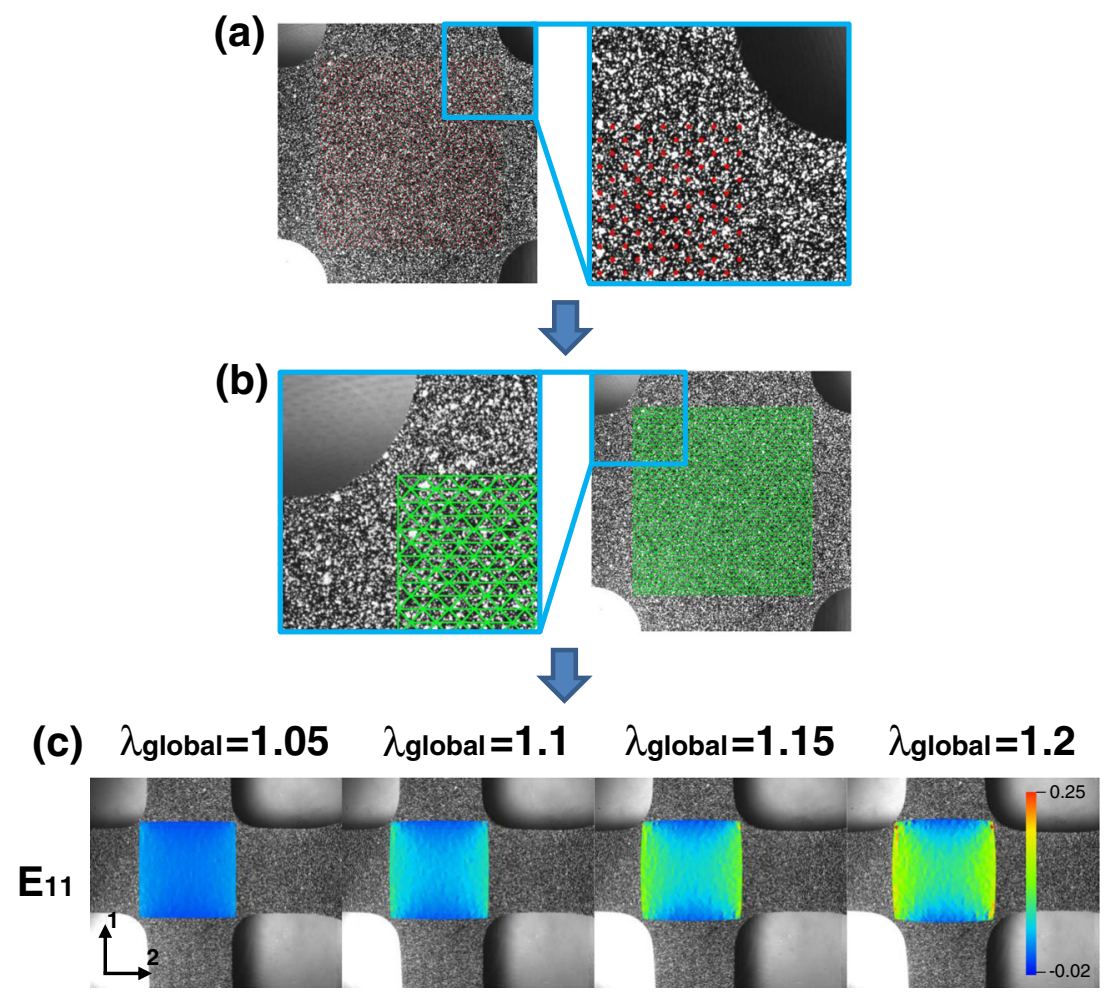

Validation of the Finite Element Models

We examined one sample for each specimen geometry. Given the test results of a sample, we obtained the optimal $\mathrm{C}_{10}$ of the sample by the inverse procedure and used the $\mathrm{C}_{10}$ to simulate the full-field deformation of the sample. Therefore, the comparisons between DIC and FEM results were based on the same sample and the same experiment; FEM results can thus be validated.

Once the $\mathrm{C}_{10}$ of each sample was determined, finite element analysis was used to simulate the full-field deformation of each sample. Here the displacement of, instead of the tensile force in, the clamped portion of each arm was specified as the boundary conditions to match the experimental protocol. Specifically, to match the global equibiaxial stretch of 1.2, the displacement of the clamped portion was set to be $5 \mathrm{~mm}$ outward. The full-field $\mathrm{E}_{11}, \mathrm{E}_{22}, \mathrm{E}_{12}, \mathrm{E}_{\mathrm{p} \text {, max }}$, and $\mathrm{E}_{\mathrm{p} \text {, max }} / \mathrm{E}_{\mathrm{p}}$, min were analyzed for comparison with the corresponding DIC results. In order to better illustrate the distribution of strains and their magnitudes, we plotted $\mathrm{E}_{11}, \mathrm{E}_{22}, \mathrm{E}_{12}$, and $\mathrm{E}_{\mathrm{p} \text {, max }}$ obtained by both DIC and FEM along centerlines and diagonal lines of the ROI. More importantly, such a plot allows direct comparisons between DIC and FEM results based on geometric locations.

Finite Element Simulation of the Strain Field

In addition to the five specimen geometries that were experimentally tested, we created a finite element model of R03125 so that comparison can be made between the general fillet and the cut-in fillet with the radius of $0.03125 \mathrm{in}$.. Finite element models of the six specimen geometries with the same material parameter $\left(\mathrm{C}_{10}=0.96 \mathrm{MPa}\right)$ were then used to estimate the influence of fillets on $E_{p}$, max as well as the strain field uniformity. Particularly, for quantification of strain field uniformity, we adopted the definition used by Eilaghi et al. [15] and measured the central, biaxially loaded area where $E_{p}, \max / E_{p}$, min is sensibly constant $\left(E_{p, \max } / E_{p}, \min \leq 1.1\right)$ and normalized it by a central square (the red outlined square in Fig. 8(a)).

\section{Estimation of Effective Transverse Length}

Finite element simulation was also used to estimate the effective transverse length required to convert tensile force at the boundary to local stress at the center; note that the effective transverse length does not equal to specimen arm width largely due to the incorporation of the fillets and non-uniform stress/strain field in the central region. In this analysis, the displacements in the clamped portions were prescribed as the boundary conditions; four global equibiaxial stretches were tested: 1.1, 1.2, 1.5, and 2. The simulated tensile force at the clamped portion and the simulated Cauchy stress and simulated local stretch ratios at the center of the specimen were recorded for calculating the effective transverse length using $L_{1}^{\text {eff }}=\widetilde{\lambda}_{2}\left(\frac{\tilde{f}_{2}}{H \tilde{\sigma}_{22}}\right)$ and $L_{2}^{\text {eff }}=\widetilde{\lambda}_{1}\left(\frac{\tilde{f}_{1}}{H \tilde{\sigma}_{11}}\right)$, where $\tilde{f}_{1}$ and $\tilde{f}_{2}$ are the simulated tensile forces in the 1 and 2 directions, 
respectively, $H$ is the undeformed thickness of the specimen, $\widetilde{\sigma}_{11}$ and $\widetilde{\sigma}_{22}$ are the simulated Cauchy stress in the 1 and 2 directions, respectively, and $\widetilde{\lambda}_{1}$ and $\widetilde{\lambda}_{2}$ are the simulated local stretch ratios in the 1 and 2 directions, respectively. Note that the simulated Cauchy stress and stretch ratios were obtained by averaging their values from four central elements. Normalized effective transverse length was presented in results, which was calculated by dividing the effective transverse length by the specimen arm width.

In addition to the cruciform geometry, we constructed a finite element model of the square geometry employed by Knezevic et al. for testing the mechanical properties of collagen gels [16]. Again, the effective transverse lengths required to convert tensile force to local stress at the center at the four global equibiaxial stretches were analyzed. We further examined the effect of reducing length of porous bars, which were treated as rigid body, in that model on the normalized effective transverse length.

\section{Results}

\section{Strain Field Estimation by Digital Image Correlation}

We focused on the ROI to evaluate the boundary effects caused by the fillets. Figure 3 shows the experimental analysis (DIC) of full-field distributions of $\mathrm{E}_{11}, \mathrm{E}_{22}, \mathrm{E}_{12}$, and $\phi$ when the specimens were each loaded equibiaxially to a global stretch of $1.2\left(\lambda_{\text {global }}=1.2\right)$; numerical simulations (FEM) of corresponding strains (but not $\phi$ ) were placed in parallel for easy comparison. The full-field distributions of $E_{11}$ for the five specimen geometries appeared to be in a similar sand-clock shape pattern; $\mathrm{E}_{11}$ decreased gradually in the 1 direction from both sides toward the center while it increased gradually in the 2 direction from both sides toward the center. The full-field distribution of $E_{22}$ for each specimen geometry showed a similar but opposite pattern with the corresponding $\mathrm{E}_{11}$. Also, the full-field distributions of $E_{12}$ for the five specimen geometries appeared to be similar. The shear strain at corners of the ROI increased as the fillet radius decreased regardless of the fillet type. The central shear strain for the five specimen geometries was negligible, indicating the reliability of our experimental results. On the other hand, $\mathrm{E}_{11}, \mathrm{E}_{22}$, and $\mathrm{E}_{12}$ in the ROI appeared to be more uniform as the fillet radius decreased regardless of the fillet type. Ideally, the specimen subjected to equibiaxial stretching should not develop local rigid-body rotation. The rigid-body rotation angle appeared to be affected by the fillet radius; particularly in areas near the corners of the ROI, the smaller fillet radius the greater rigidbody rotation angle regardless of the fillet type. Also, cut-in fillets developed more of rigid-body rotation than general fillets. The central rigid-body rotation angle for the five specimen geometries was found to be within $3.75^{\circ}$, again indicating the reliability of our experimental results.

Figure 4 shows that the greatest value of $E_{p, \text { max }}$ for the five specimen geometries occurred at the edge of fillets while it had the minimum at the center of the specimen. Specifically, the smaller fillet radius the greater $E_{p}$, max regardless of the fillet type. Figure 5 shows that $E_{p, \max } / E_{p, \text { min }}$ equal to one at the center. The strain field uniformity decreased with increasing fillet radius regardless of the fillet type. Note particularly that cut-in fillets provided better strain field uniformity than general fillets given the same fillet radius.

We found that as the specimen was gradually stretched, $\mathrm{E}_{11}$ and $\mathrm{E}_{22}$ increased at the center while $\mathrm{E}_{12}$ remained negligible at the center but increased at the corners (Supplemental Figure 2).

Constitutive Modeling of Cruciform Rubber Specimens with Various Fillets

We found that slightly different values of the $\mathrm{C}_{10}$ were reached for the samples (R2500: $0.951 \mathrm{MPa}, \mathrm{R} 1250: 0.978 \mathrm{MPa}$, R0625: $0960 \mathrm{MPa}$, In_03125: 0. $935 \mathrm{MPa}$, and In 0625: $0.972 \mathrm{MPa}$ ). Note again that we simulated full-field deformation of each sample with its corresponding $\mathrm{C}_{10}$.

\section{Strain Field Estimation by Finite Element Analysis}

Although shown in a similar but slightly different color scales, the FEM results appeared to be in good agreement with the DIC results (Figs. 3, 4, and 5); the similarity between FEM and DIC validated the finite element models. Note that the boundary conditions used for the simulation does not generate rigid-body rotation (i.e., $\mathrm{F}_{12}=\mathrm{F}_{21}$ ) and thus no simulated fullfield rigid-body rotation angle was shown.

Figure 6 shows $\mathrm{E}_{11}, \mathrm{E}_{22}, \mathrm{E}_{12}$, and $\mathrm{E}_{\mathrm{p} \text {, max }}$ obtained by both DIC and FEM along centerlines and diagonal lines of the ROI for the five specimen geometries. Again, the FEM results appeared to fit the DIC results well. For the five specimen geometries $\mathrm{E}_{11}$ along the horizontal centerline had the minimum at the center of the specimen while $\mathrm{E}_{11}$ along the vertical centerline had the maximum at the center. $E_{22}$ for each specimen geometry showed a similar but opposite pattern with the corresponding $\mathrm{E}_{11}$. Specifically, the $\mathrm{E}_{11}$ at the center of the specimen increased gradually from R2500 to In 0625 ; recall that the specimens were each loaded equibiaxially to a global stretch of 1.2. No obvious change in $E_{12}$ was observed in the centerlines. $\mathrm{E}_{12}$, however, had the maximum or the minimum at the two ends of the diagonal lines. The minimum of $E_{p, \max }$ located at the center of the specimen and its value increased from R2500 to In 06125. Generally, we found that DIC results were in good agreement with FEA results at the center of the specimen. The difference between DIC and FEM results 
Fig. 3 The full-field distributions of strains $\left(E_{11}, E_{22}, E_{12}\right)$ and rigidbody rotation angle $(\phi)$ in the central region analyzed experimentally by digital image correlation (DIC) and simulated numerically by finite element method (FEM) for the five specimen geometries. The specimens were each loaded to a global equibiaxial stretch of 1.2 . Note that the color scale for DIC and that for FEM are similar but slightly different

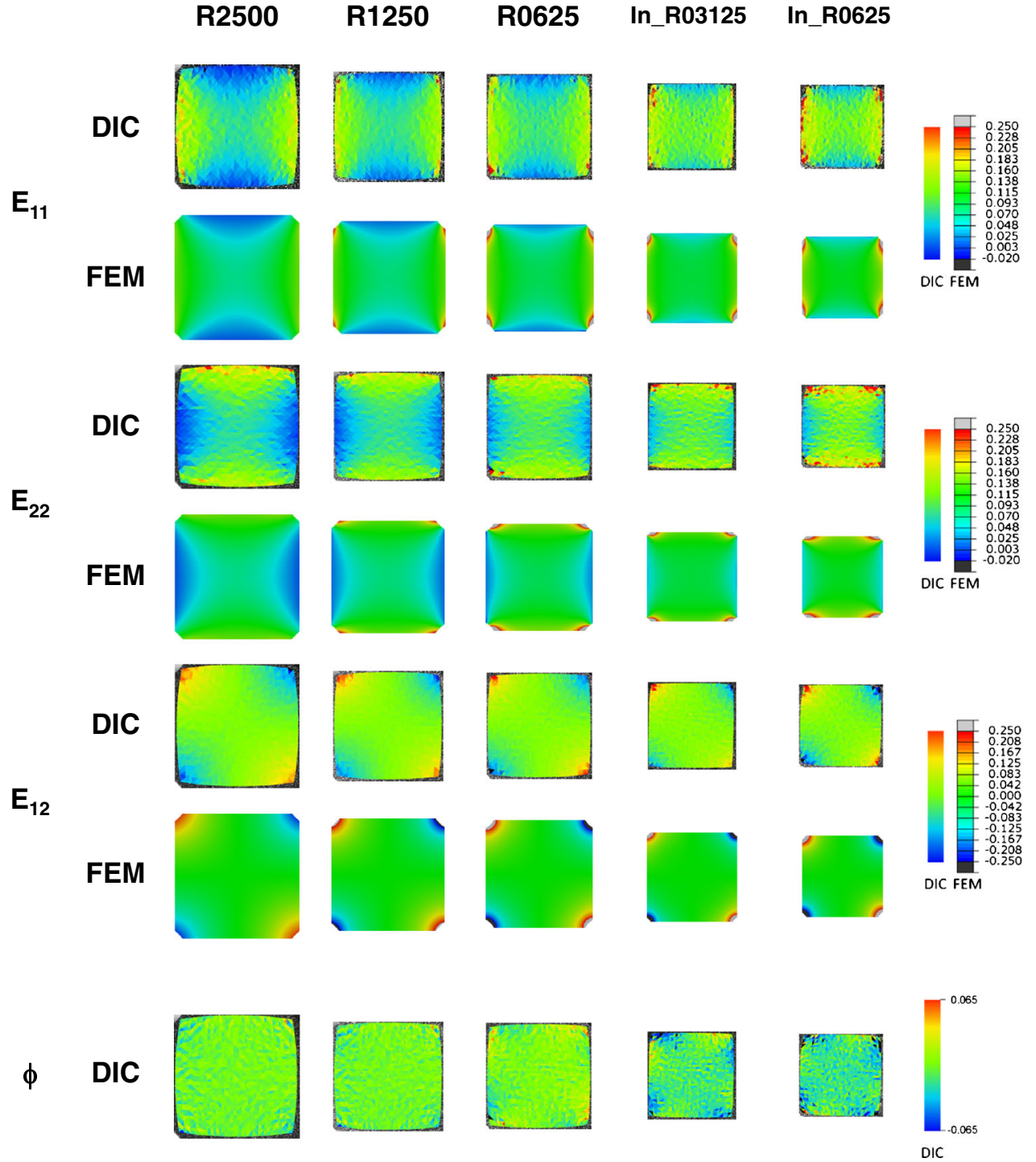

perpendicular arms. Figure 7 shows that the value of $E_{p}$, max decreased with increasing fillet radius. Note, particularly, that the values of $E_{p, \max }$ were similar for the two fillet types given the same fillet radius. were more notable at the two ends of centerlines or diagonal lines.

The finite element simulations showed that $\mathrm{E}_{\mathrm{p}, \max }$ for the five specimen geometries occurred at the intersections of

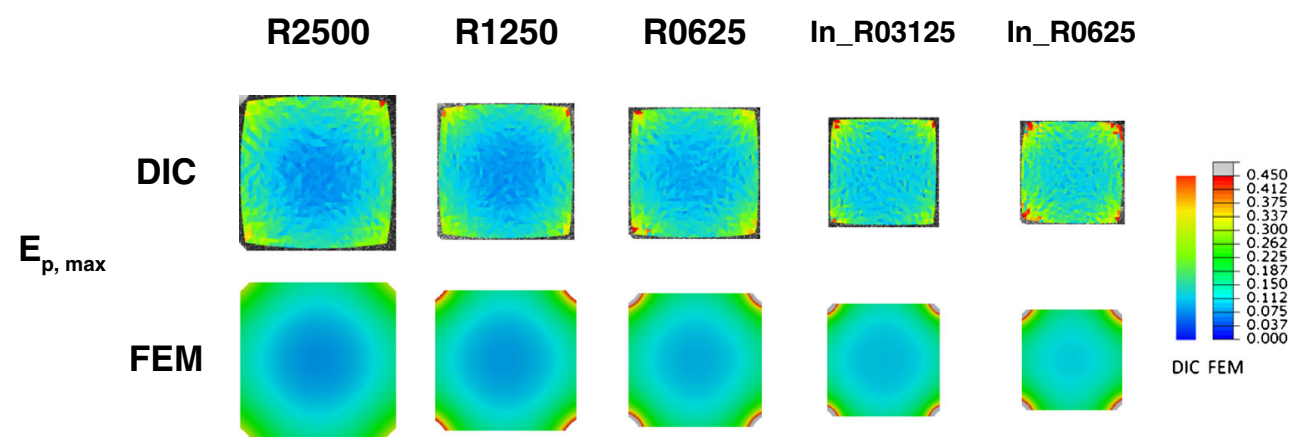

Fig. 4 The full-field distributions of the maximum principal strain $\left(E_{p, m a x}\right)$ in the central region analyzed experimentally by digital image correlation (DIC) and simulated numerically by finite element method (FEM) for the five specimen geometries. The specimens were each loaded to a global equibiaxial stretch of 1.2. Note that the color scale for DIC and that for FEM are similar but slightly different 


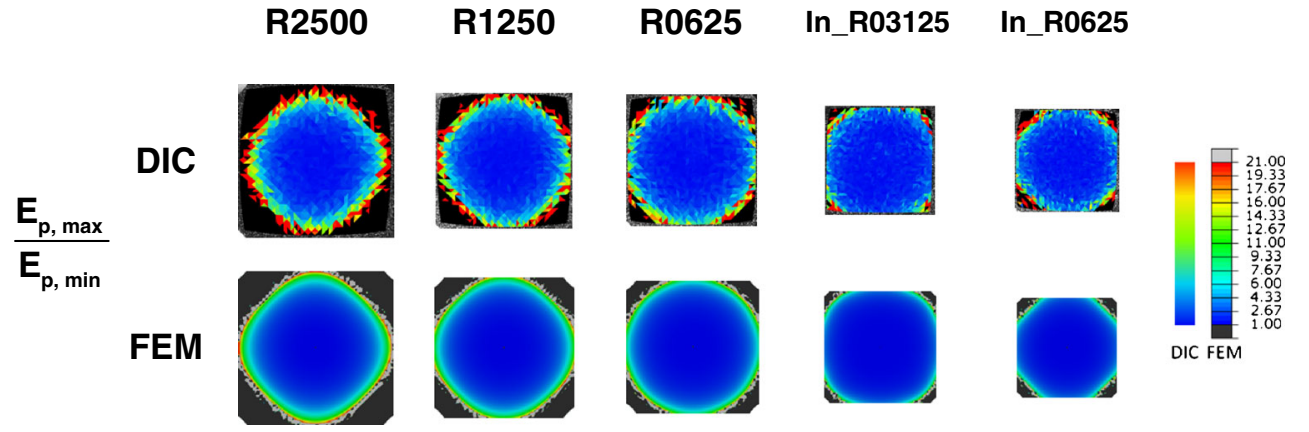

Fig. 5 The full-field distributions of the ratio of principal strains $\left(E_{p, \max } / E_{p, \min }\right)$ in the central region analyzed experimentally by digital image correlation (DIC) and simulated numerically by finite element method (FEM) for the five specimen geometries. The specimens were each loaded to a global equibiaxial stretch of 1.2. Note that the color scale for DIC and that for FEM are similar but slightly different

The uniform strain area, in which $\mathrm{E}_{\mathrm{p}, \max } / \mathrm{E}_{\mathrm{p} \text {, min }}$ is less than 1.10, increased as the fillet radius decreased (Fig. 8(a)). If the area is normalized by the red outlined square in (Fig. 8(a)), it appeared that cut-in fillets allowed greater normalized uniform strain area than general fillets given the same fillet radius (Fig. 8(b)). Note that this comparison was based on the same global equibiaxial stretch; although not shown, we found that the strain field uniformity decreased as the global stretch increased.

Figure 9 shows that the normalized effective transverse length increased for general fillets but decreased for cut-in fillets as the fillet radius increased. The normalized effective transverse length for each specimen geometry was estimated with three different mechanical properties ranging from soft $\left(\mathrm{C}_{10}=0.5 \mathrm{MPa}\right)$, medium $\left(\mathrm{C}_{10}=0.96 \mathrm{MPa}\right)$ to stiff $\left(\mathrm{C}_{10}\right.$ $=.5 \mathrm{MPa}$ ). The normalized effective transverse length appeared to approach the minimum for general fillets with decreasing fillet radius. The normalized effective transverse length nearly reached 1.0 for cut-in fillets with the fillet radius of $0.0625 \mathrm{in}$.. For general fillets with larger radii, the normalized effective transverse length decrease initially and then increased as the global stretch increased while for cut-in fillets and general fillets with smaller radii the normalized effective transverse length increased as the global stretch increased. It appeared that the normalized effective transverse length for each specimen geometry varied with the global stretch but was not affected significantly by the mechanical properties examined. In general, however, the normalized effective transverse length for each specimen geometry remained relatively constant if the specimen was not significantly deformed (within a global equibiaxial stretch of 1.2).

Finite element simulations illustrated that the square specimen with shorter bars had significantly lower $E_{p, \text { max }}$ at the corners of the specimen compared to that with longer bars when both were loaded equibiaxially to a global stretch of 1.2 (Fig. 10(a)). It was found that the specimen with longer bars deformed only at the corners leaving the central region almost undeformed when the tensile forces that the specimen with shorter bars was subjected to in the previous setting were assigned as the boundary conditions (Fig. 10(b)). Figure 11 shows that reducing length of porous bar significantly reduced the normalized effective transverse length. Note that as the global stretch increased the normalized effective transverse length increased for the specimen with either longer or shorter bars.

\section{Discussion}

In the fields of metal and plastic testing, cruciform specimens are usually used for planar biaxial mechanical testing. For instance, the design of cruciform specimen for composite material testing has been investigated intensively [17-19]. Although most planar biaxial mechanical properties of soft tissues are tested with square specimens loaded via arrays of sutures, extremely soft tissues prohibit the use of suture attachment and may only be tested using cruciform specimens. Unlike metals or plastics, however, loaded soft tissues usually undergo large deformation and associated shape changes of specimen may have profound boundary effects on measured stiffness data.

Not much has been done, however, in the field of soft tissue testing with respect to cruciform specimen design. Sun et al. demonstrated using finite element methods that clamp-based methods have substantially stronger boundary effects in comparison to suture-based methods, which results in lower central stress in clamped specimens than in suture-attached specimens under the same equibiaxial loading [11]. For clamped cruciform specimens, increasing specimen arm length alleviates the clamp-related boundary effects. Note that our cruciform specimens had arms $200 \%$ of the length of the central square. Note also that the finite element models of their cruciform specimens contain no fillets [11], which may result in singular points at the intersections of perpendicular arms and lead to extreme stress at these areas. Waldman and Lee experimentally tested cruciform specimens with different specimen arm lengths from fish skin, bovine pericardium and model textile laminates, and found similarly that the apparent mechanical properties of the materials is dependent 
R2500
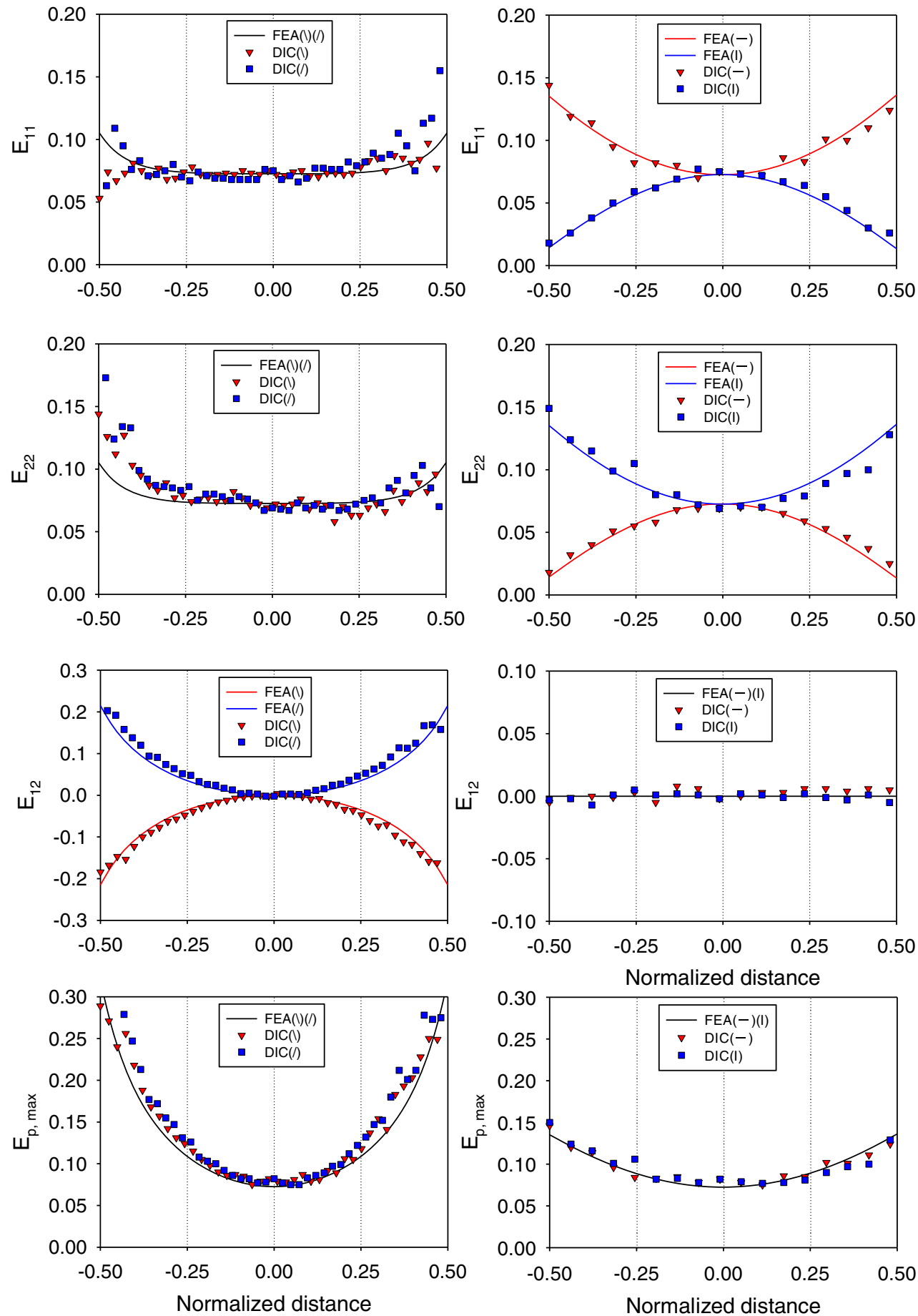

Fig. 6 Comparisons of strains analyzed experimentally (DIC) and simulated numerically (FEA) along the diagonal lines ( $(, /)$ and the horizontal and vertical centerlines $(-, \mid)$ of the region of interest for the five specimen geometries. The specimens were each loaded to a global equibiaxial stretch of 1.2 . The symbols represent the results of DIC and the lines the results of FEA

on the specimen arm length [12]. The geometric factor of interest in these studies is specimen arm length. Little is known about how the fillets in a cruciform specimen affect the full-field strain distribution as well as the stiffness data generated from a test. Very recently, Bell et al. used finite element analysis to optimize the cruciform specimen geometry aiming at maximizing stresses within the central region while diminishing stress concentrations at arm attachment points [10]. The radius of the attachment arm junction was the only design parameter; that is, other key geometric factors 
R1250
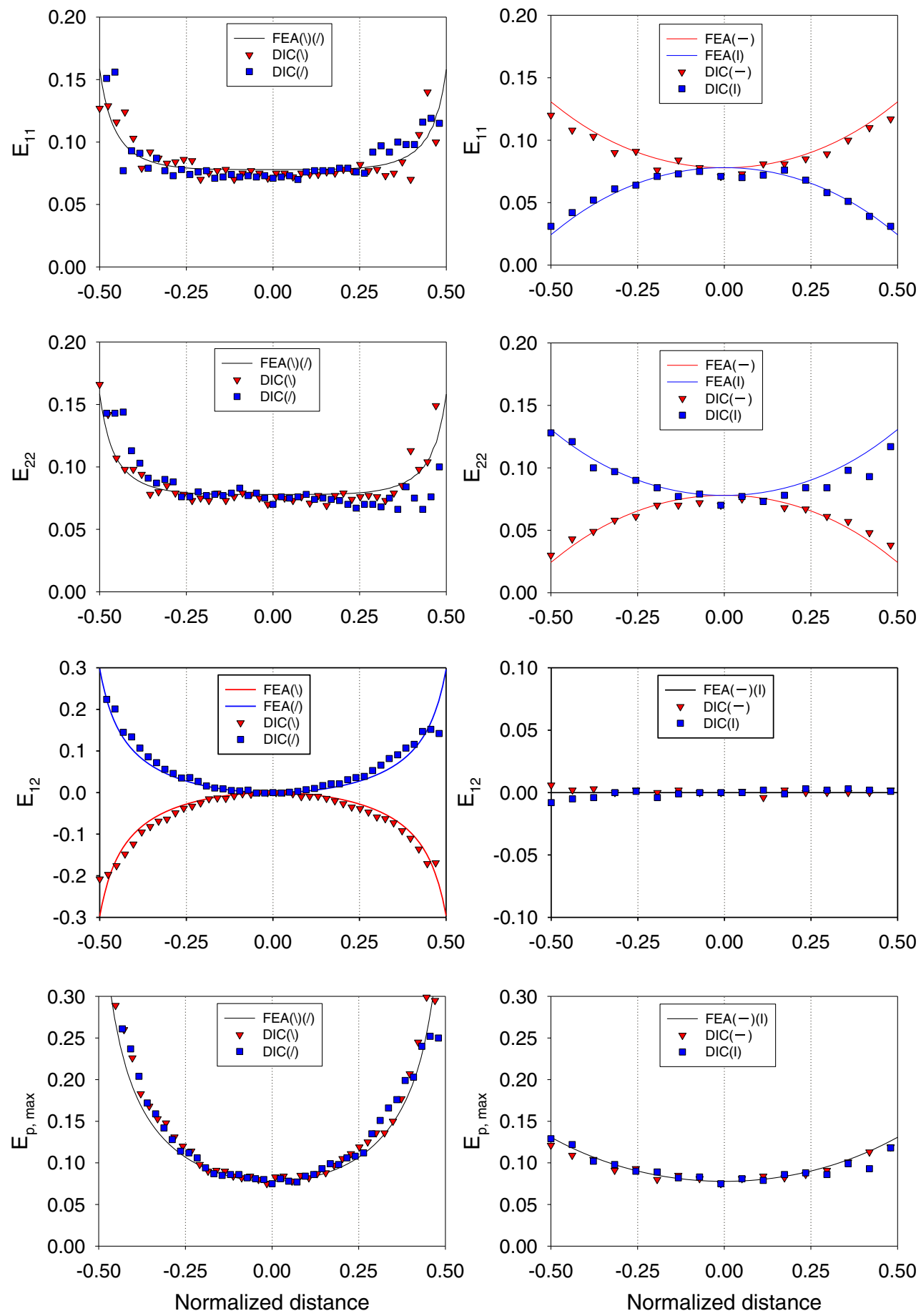

Fig. 6 (continued)

such as specimen arm width are dependent on the radius and cannot be controlled. Furthermore, analysis in apparent mechanical properties was not conducted in their study.

The design criteria for the optimal geometry of cruciform specimens should include, at least, the following: (1) Shear strain and rigid body rotation angle must be negligible in the central region of the specimen. For all of the specimens examined in this study, both the shear strain and the rigidbody rotation angle in the central region were negligible throughout the test, suggesting that the experimental results are reliable. Note that it is important not to infer shear information from planar biaxial tests because of the inability to impose defined shear stresses [20]. (2) The value of $E_{p}$, max must be within the failure limit of tested materials during 
R0625
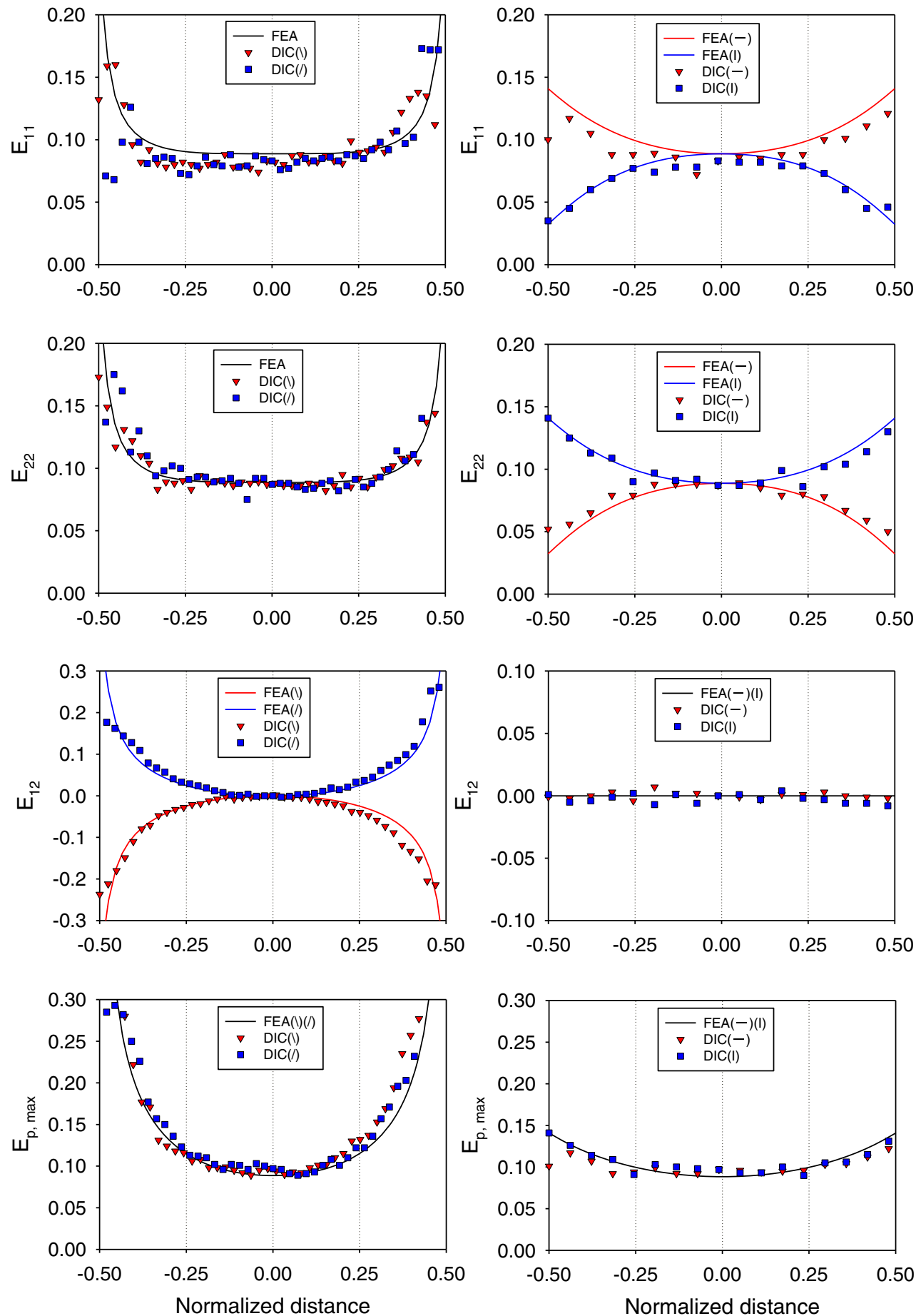

Fig. 6 (continued)

testing. Our results suggested that potential failure at the intersections of perpendicular arms can be avoided by increasing the fillet radius regardless of the fillet type. Note, however, that cruciform specimens when stretched excessively might fail at arms if not fail at the intersection of arms. That is, the range of deformation in the central region is inherently limited. (3) The strain field in the central region must be sufficiently uniform. The issue of strain field uniformity is more of a concern if strain gauges or extensometers are used for strain measurements. Even for some noncontact optical approach 
In_R03125
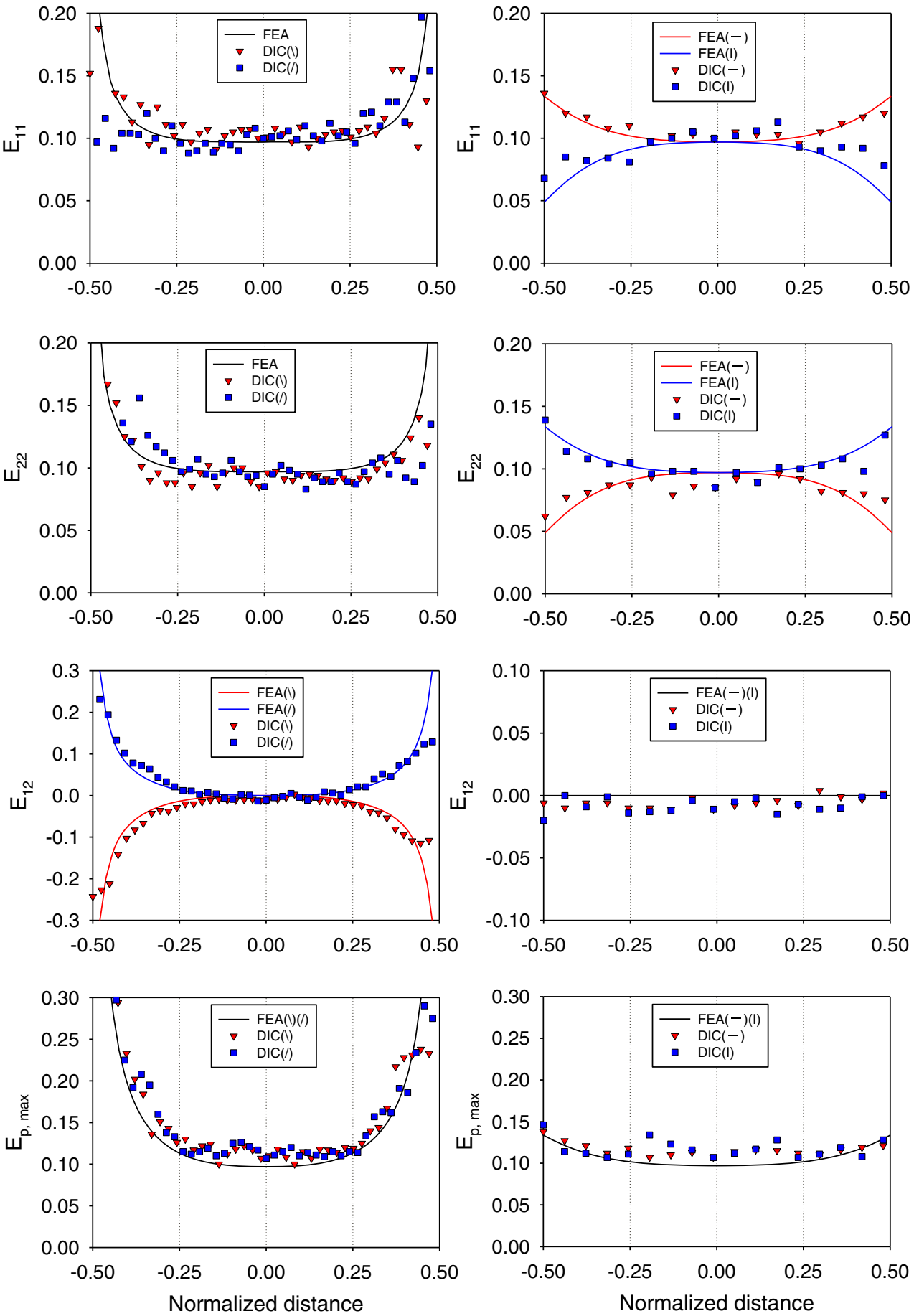

Fig. 6 (continued)

(e.g., video-dimension analyzer), the strain field uniformity in the measuring area is also important. Our results suggested using cut-in fillets for better strain field uniformity given the same fillet radius. (4) At the center of the specimen where stiffness data are collected, the corresponding stress can be calculated without ambiguity. The last criterion is affected mainly by the ill-defined cross-sectional area of the central region as well as the non-uniform stress/strain field in the central region; the latter is further degraded by the incorporation of fillets. It is therefore necessary to determine the effective transverse length and investigate how the fillets affect the effective transverse length. Interestingly we found that 
In_R0625
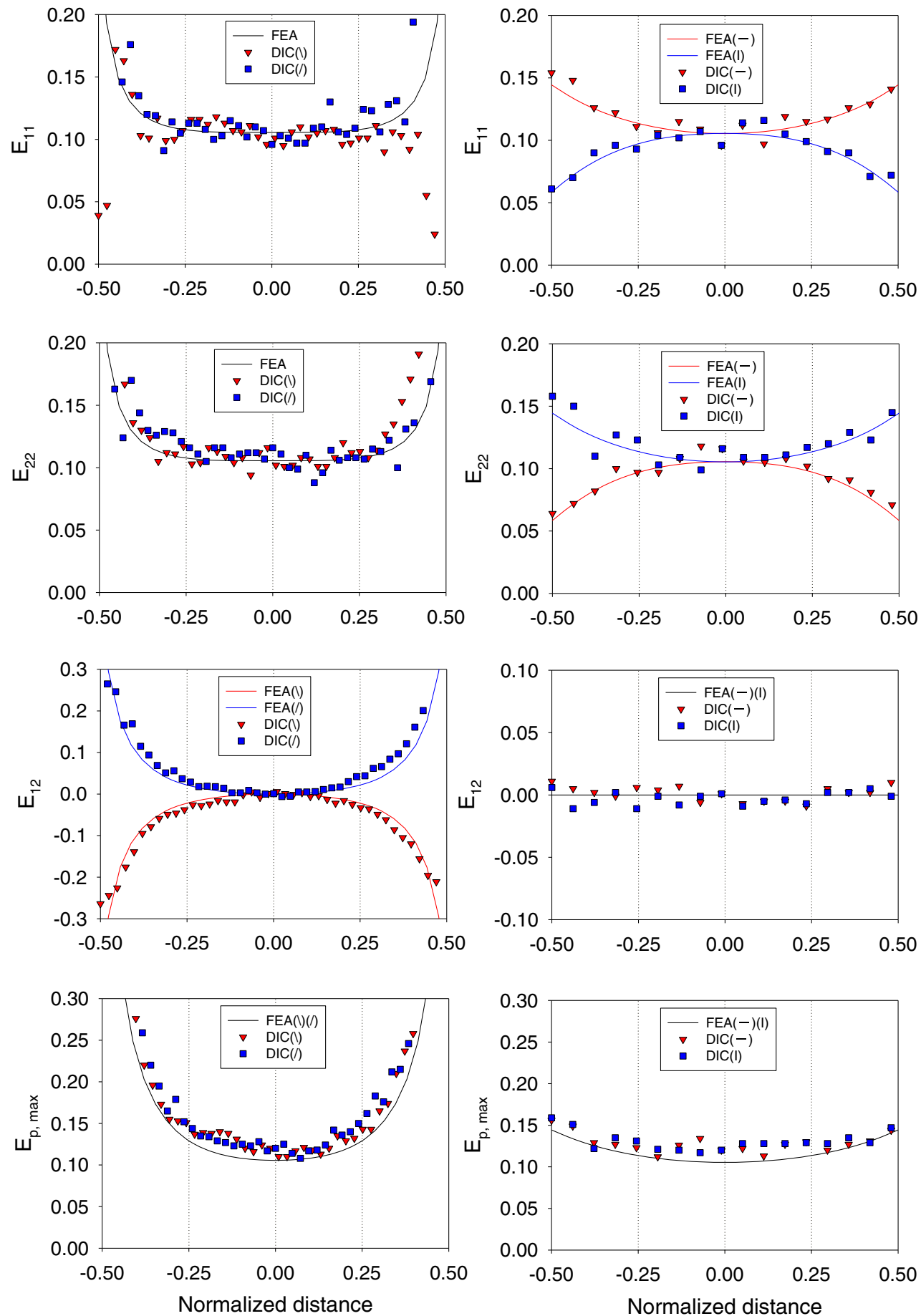

Fig. 6 (continued)

effective transverse length for each specimen geometry remained relatively constant if the specimen was not excessively deformed (i.e., global equibiaxial stretch $\leq$ 1.2). As the deformation becomes significant, which is not uncommon for soft tissues, the shape of the specimen as well as the uniformity of strain field change; both of which may contribute to the increased effective transverse length. Note that the effective transverse length for all the specimens with general fillets was found to be greater than the specimen arm width; that is, stress will be over-estimated if the arm width is used to calculate stress. 


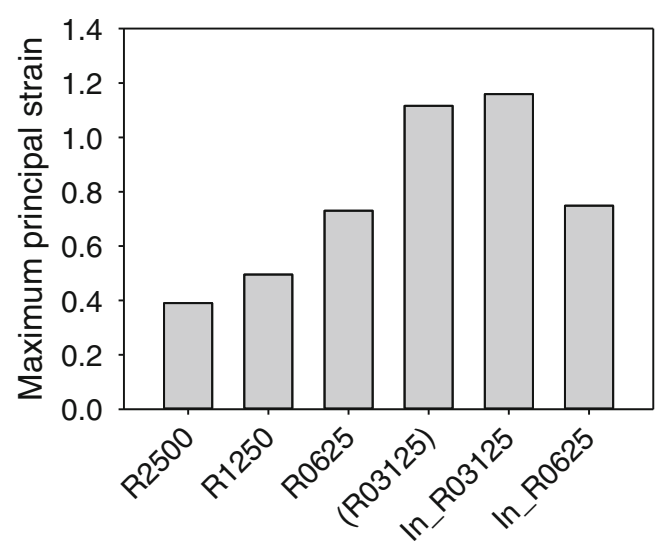

Fig. 7 Comparison of the greatest value of $E_{p, \max }$ obtained by FEM for the six specimen geometries. Note that R03125 was not experimentally examined. Global equibiaxial stretch of 1.2 was assigned as the boundary conditions

Generally a full-field strain measurement involves determination of motions of patterns/markers by DIC and estimation of strain field based on results of DIC although some DIC algorithms such as Newton-Raphson or Levenberg-Marquart algorithms directly generate the displacement gradients (i.e., strains) [21]. In this study, a custom code in LabVIEW was written for DIC and it appeared to provide satisfactory motion data of speckles with sub-pixel accuracy. Regarding to strain field estimation, we meshed the ROI with triangular elements and specifically used a linear interpolation function to smoothen displacement field within each element, resulting in a constant deformation gradient within the element. This approach is straightforward and fast but limited to analysis of a relatively smooth strain field; the error of strains may be significant in areas with steep deformation gradient. That is, within the ROI the deformation gradient as well as Green strain, principal strains, and rigid-body rotation angle near the fillets may not be as accurate as that in the central region. This may in part explain the discrepancy between DIC and FEM at these areas. Nevertheless, the code allowed determining the full-field strain rapidly and real-time evaluation of strain distribution was possible; this grants the capability of strain-based controlled mechanical testing. Essentially, the maximum number of elements analyzed by DIC is limited by the capability of the computer.

The purpose of full-field strain measurement by DIC in this study was to evaluate the strain distribution in the central region and validate our finite element simulations. The analysis of DIC was restricted to the rectangular ROI. DIC inherently cannot be performed at the very edge of fillets where the greatest value of $E_{p, \max }$ is expected to occur. Therefore, we relied on FEM to estimate $E_{p, \text { max }}$ at these areas.

The slight variations in $\mathrm{C}_{10}$ for each specimen geometry was expected considering the experimental errors, interspecimen variations, and that the Neo-Hookean model is far from perfect for describing the mechanical behavior of the rubber. We did not intend to find a single material parameter for the five specimen geometries, which might be done by grouping all the experimental and simulated data of the five specimen geometries in a single objective function. The purpose of using different $\mathrm{C}_{10}$, as pointed out in the Methods, was to compare DIC and FEM results on the basis of the same sample and the same experiment and thus FEM results can be validated properly. Nevertheless, we used a single $C_{10}$ $\left(0.96 \mathrm{MPa}\right.$ ) in analyzing $\mathrm{E}_{\mathrm{p} \text {, max }}$ and $\mathrm{E}_{\mathrm{p}, \max } / \mathrm{E}_{\mathrm{p} \text {, min }}$ (strain field uniformity index) so that the comparisons are totally based on specimen geometries without the influence of material behavior.

Standard uniaxial tensile testing allows generating stressstrain data that can also be used to determine the $\mathrm{C}_{10}$ of the rubber. We thus prepared rectangular specimens $(50 \mathrm{~mm} \times$ $5 \mathrm{~mm} \times 0.3 \mathrm{~mm}$ ) from the rubber sheets for uniaxial tensile tests. We found that the $\mathrm{C}_{10}$ of the Neo-Hookean model depended on the range of the data used for fitting (Supplemental Figure 3). Nevertheless, the values of the $C_{10}$ were comparable to those obtained in this study. Note that a constitutive model with material parameters obtained by fitting data (a)

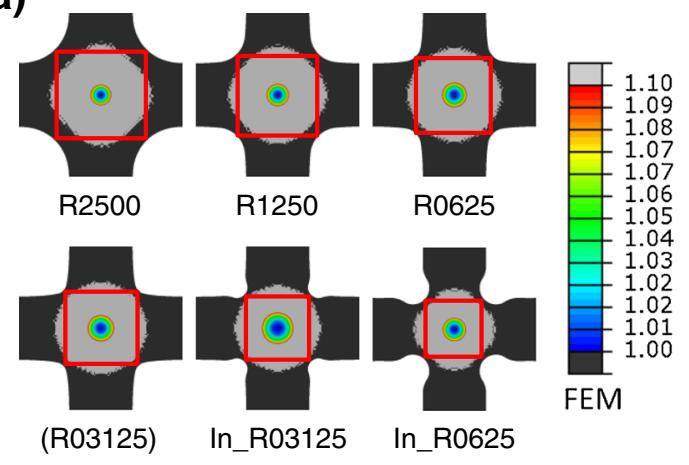

(b)

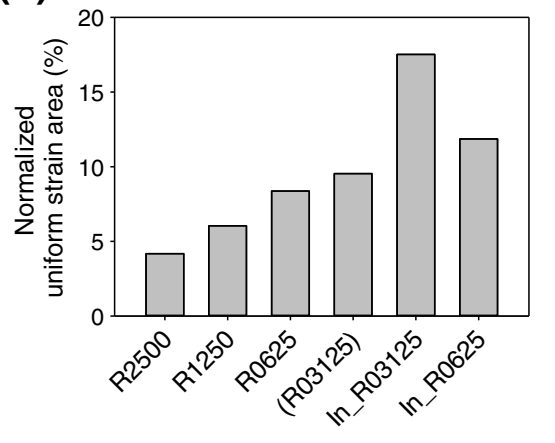

Fig. 8 Comparison of the normalized uniform strain area obtained by FEM for the six specimen geometries. (a) FEM results of the ratio of principal strain. The normalized uniform strain area is the area in which the ratio is less than 1.1 normalized by the red square area for each specimen geometry (b). Note that (R03125) was not experimentally examined. Global equibiaxial stretch of 1.2 was assigned as the boundary conditions. Note that the gray area indicates $\mathrm{E}_{\mathrm{p}, \max } / \mathrm{E}_{\mathrm{p}, \min }>1.10$ and black area $\mathrm{E}_{\mathrm{p}, \max } / \mathrm{E}_{\mathrm{p}, \min }<1.00$ 
Fig. 9 The normalized effective transverse length calculated based on four different prescribed boundary conditions (global equibiaxial stretch $=1.1,1.2$, 1.5 , and 2) for the six specimen geometries (a): R2500, (b): R1250, (c): R0625, (d): R03125, (e): In_R03125, and (f): In_R0625). The number in parentheses indicates the simulated local stretch ratio at the center of the specimen corresponding to the global stretch. Note that (R03125) was not experimentally examined (a)

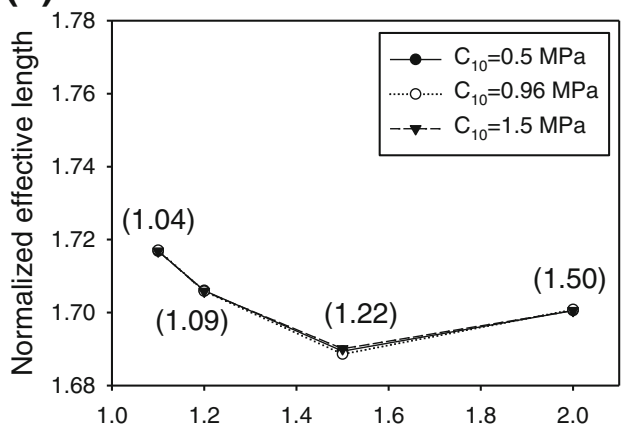

(b)

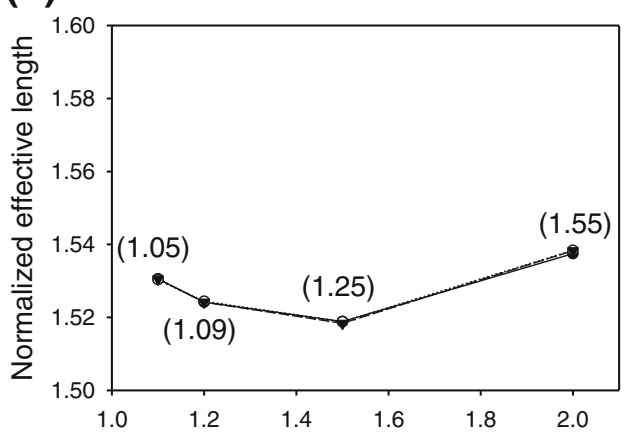

(c)

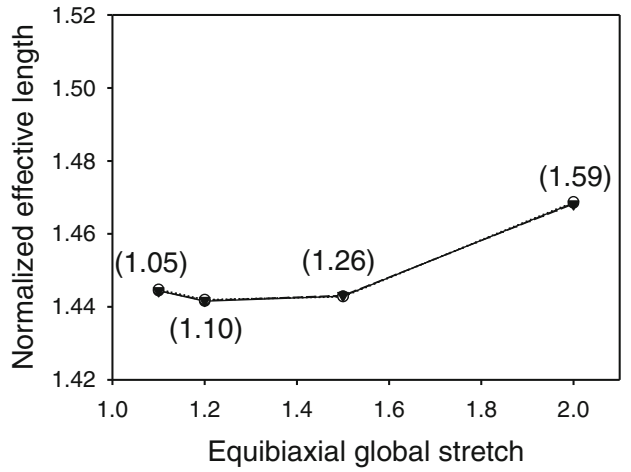

(d)

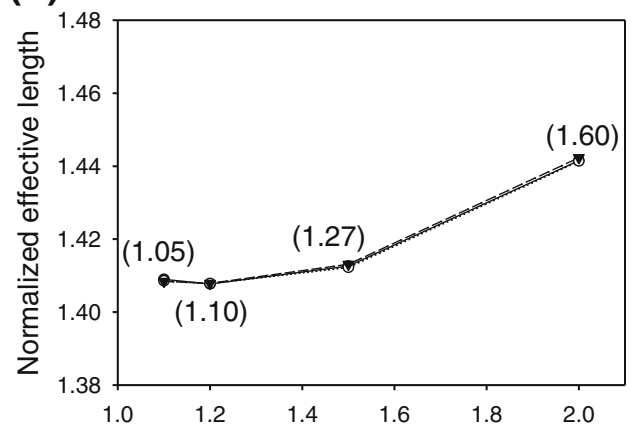

(e)

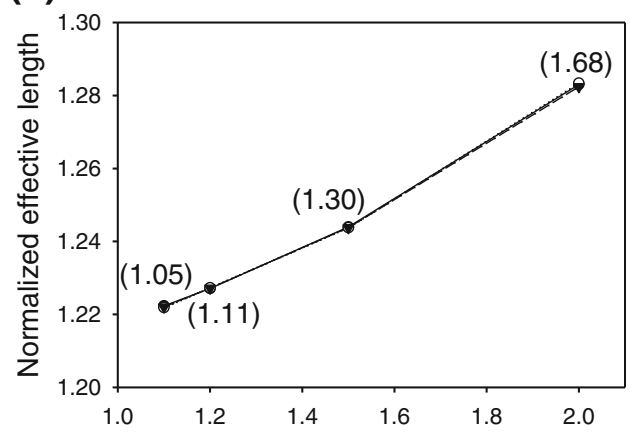

(f)

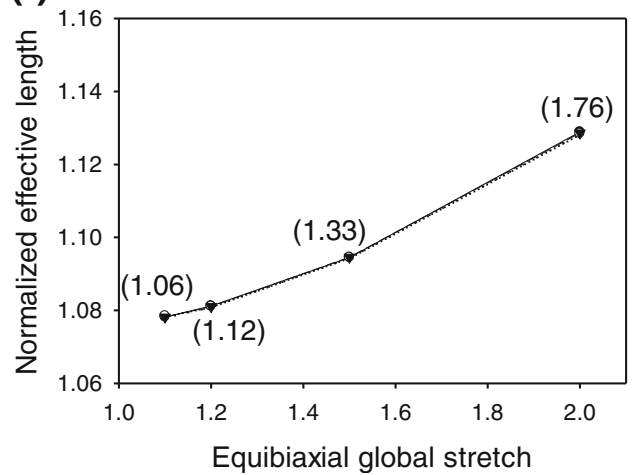

of uniaxial tensile tests of a material may not predict well the biaxial mechanical behavior of that material. Beda provided such an example in his study of rubber [22].
Other constitutive models for rubber such as the MooneyRivlin or Ogden strain energy function may serve better than the Neo-Hookean model. Note again that in this study the $\mathrm{C}_{10}$ (a)

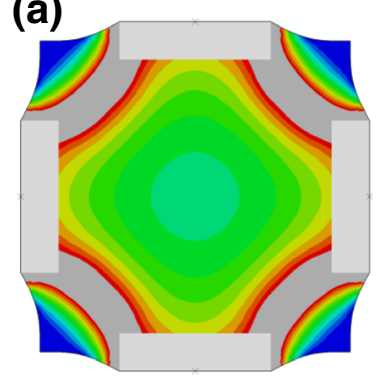

(b)

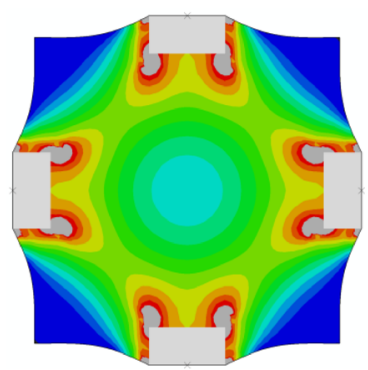

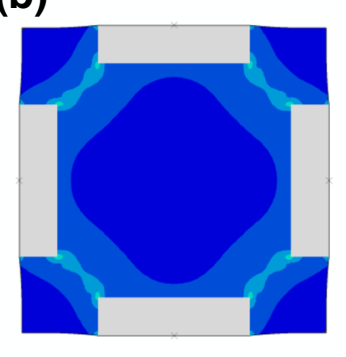

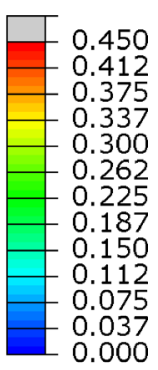

Fig. 10 FEM results of the $E_{p \text {, max }}$ for two square specimen geometries; the length of the shorter bar is half of that of the longer bar. Global equibiaxial stretch of 1.2 was assigned as the boundary conditions ( $(\mathbf{a}$ and $\mathbf{b})$. Equibiaxial tensile force of $4.8 \mathrm{~N}$ was assigned as the boundary conditions $(\mathbf{b}$ and $\mathbf{c}$ ). Note that the gray area indicates $\mathrm{E}_{\mathrm{p}, \max }>0.45$ 


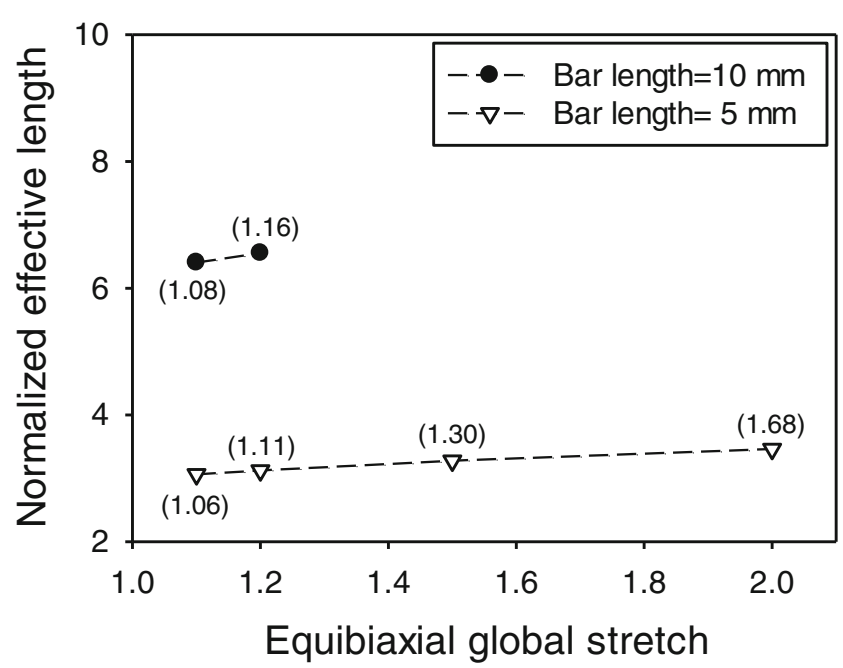

Fig. 11 The normalized effective transverse length calculated based on four different prescribed boundary conditions (global equibiaxial stretch $=$ $1.1,1.2,1.5$, and 2) for the two square specimen geometries. The number in parentheses indicates the simulated local stretch ratio at the center of the specimen corresponding to the global stretch. Note that FEM failed because of excessively distorted elements when the square specimen with longer bars was loaded to global equibiaxial stretches of 1.5 and 2

of a sample can be obtained manually because only one material parameter is involved in the Neo-Hookean model and the optimization algorithm in the inverse procedure compares only strains at the center of the specimen (not the full-field strain). If the constitutive model containing more than one material parameter is used, a nonlinear regression algorithm will be needed and particularly the material parameters obtained might not be unique [23]. With respect to characterizing materials using full-field strain data, a sophisticated optimization algorithm similar to the one developed by Lecompte D. et al. will be needed [24]. Note also that others have used the boundary element method [25] or the virtual fields method [26] besides the finite element method [24] in extracting material parameters of constitutive models from full-field strain data.

The full-field strain distributions obtained by both DIC and FEM were essentially derived from the deformation gradient of each element. That is, large deformation of the specimen was taken into account in both DIC and FEM analyses. This is particularly important as loaded soft tissues usually undergo large deformation and linear elasticity could lead to inaccurate results. For example, rigid-body rotation does not affect the calculation of Green strain. Note that we calculated rigid-body rotation angle also from the deformation gradient. The rigidbody rotation angle is not the angle change in the definition of linear shear strain; shear strain at large deformation includes contributions from both angle change and extension [27].

In addition to the cruciform geometry, we constructed a finite element model of the square geometry employed by Knezevic et al. for testing the mechanical properties of collagen gels [16]. The existence of relatively rigid bars appeared to prevent free edge expansion in each of two lateral directions thus could lead to artificially high stiffness. The problem may be resolved by reducing the length of the bars; the square specimen with shorter bars essentially behaves like a clamped cruciform specimen.

Instead of cell-seeded collagen gels, rubber specimens were prepared and tested in this study. Rubber is considered to be an incompressible, isotropic, elastic material and thus the results of analysis can be interpreted easily. The constitutive models for rubber are well-established and can be implemented in finite element analysis with little effort. Furthermore, the mechanical behavior of rubber, unlike that of general soft tissues which is subjected to biological variations, is relatively consistent between specimens. Nevertheless, testing on anisotropic soft materials/ tissues, which is beyond the scope of this study, may provide more insights on cruciform specimen design and warrants further investigation.

\section{Conclusion}

The Neo-Hookean model fit the experimental data reasonably well and the associated finite element models of the specimen geometries were validated well by digital image correlation. The results of finite element simulation indicated that the greatest value of the maximum principal strain as well as the strain field uniformity in the central region was reduced by increasing the fillet radius regardless of the fillet type, that the use of cut-in fillets provided greater strain field uniformity compared with general fillets given the same fillet radius, and that effective transverse length for each specimen geometry remained relatively constant if the specimen was not excessively deformed (i.e., global equibiaxial stretch $\leq 1.2$ ). We suggest using cut-in fillets at the intersections of perpendicular arms when preparing cruciform specimens for testing extremely soft tissues.

Acknowledgments The authors would like to thank Dr. Chih-Han Chang for his advice on finite element simulation. Technical assistance provided by Mei-Xuan Wang was gratefully acknowledged. This research was supported by a grant from the National Science Council in Taiwan (NSC-97-2218-E-006-289-MY2).

\section{Appendix A}

A linear interpolation function for a triangular element was used, which is defined as $N_{i}=\frac{a_{i}+b_{i} X_{1}+c_{i} X_{2}}{2 \cdot \text { Area }} ; i=1,2,3$,

where

Area $=\frac{1}{2} \operatorname{det}\left|\begin{array}{rrr}1 & X_{1}^{1} & X_{2}^{1} \\ 1 & X_{1}^{2} & X_{2}^{2} \\ 1 & X_{1}^{3} & X_{2}^{3}\end{array}\right|=\frac{1}{2}\left(X_{1}^{2} X_{2}^{3}+X_{1}^{3} X_{2}^{1}+X_{1}^{1} X_{2}^{2}-\right.$

$\left.X_{1}^{2} X_{2}^{1}-X_{1}^{3} X_{2}^{2}-X_{1}^{1} X_{2}^{3}\right), a_{1}=X_{1}^{2} X_{2}^{3}-X_{1}^{3} X_{2}^{2}, a_{2}=X_{1}^{3} X_{2}^{1}-X_{1}^{1}$ $X_{2}^{3}, a_{3}=X_{1}^{1} X_{2}^{2}-X_{1}^{2} X_{2}^{1}, b_{1}=X_{2}^{2}-X_{2}^{3}, b_{2}=X_{2}^{3}-X_{2}^{1}, b_{3}=$ $X_{2}^{1}-X_{2}^{2}, c_{1}=X_{1}^{3}-X_{1}^{2}, c_{2}=X_{1}^{1}-X_{1}^{3}, c_{3}=X_{1}^{2}-X_{1}^{1}$ and, $X_{1}$ 
and $X_{2}$ are reference coordinates; the subscript indicates coordinate in the 1 or 2 direction and the superscript indicates the three vertices of the triangular element. With the interpolation function, the position vector $\mathbf{x}=\left(x_{1} x_{2}\right)$ of a particle within the triangular element in a deformed configuration was approximated in terms of the position vectors of the three vertices of the triangular element in the deformed configuration $\mathbf{x}^{j}=\left(x_{1}{ }^{j} x_{2}{ }^{j}\right) ; j=1,2,3$, as follows.

$x_{1}\left(X_{1}, X_{2}\right) \cong N_{1} x_{1}^{1}+N_{2} x_{1}^{2}+N_{3} x_{1}^{3}$ $x_{2}\left(X_{1}, X_{2}\right) \cong N_{1} x_{2}^{1}+N_{2} x_{2}^{2}+N_{3} x_{2}^{3}$

Because the interpolation functioin is a function of reference coordinates, the position vector is also a function of reference coordinates. Then the components of 2dimentional deformation gradient $\mathbf{F}_{2 \mathrm{D}}\left(\mathbf{F}=\frac{d \mathbf{x}}{d \mathbf{X}}\right)$ were calculated using

$$
\begin{aligned}
& F_{11}=\frac{d x_{1}}{d X_{1}}=\frac{b_{1}}{2 \cdot \text { Area }} x_{1}^{1}+\frac{b_{2}}{2 \cdot \text { Area }} x_{1}^{2}+\frac{b_{3}}{2 \cdot \text { Area }} x_{1}^{3}, \\
& F_{12}=\frac{d x_{1}}{d X_{2}}=\frac{c_{1}}{2 \cdot \text { Area }} x_{1}^{1}+\frac{c_{2}}{2 \cdot \text { Area }} x_{1}^{2}+\frac{c_{3}}{2 \cdot \text { Area }} x_{1}^{3}, \\
& F_{21}=\frac{d x_{2}}{d X_{1}}=\frac{b_{1}}{2 \cdot \text { Area }} x_{2}^{1}+\frac{b_{2}}{2 \cdot \text { Area }} x_{2}^{2}+\frac{b_{3}}{2 \cdot \text { Area }} x_{2}^{3}, \\
& F_{22}=\frac{d x_{2}}{d X_{2}}=\frac{c_{1}}{2 \cdot \text { Area }} x_{2}^{1}+\frac{c_{2}}{2 \cdot \text { Area }} x_{2}^{2}+\frac{c_{3}}{2 \cdot \text { Area }} x_{2}^{3} .
\end{aligned}
$$

The deformation gradient is found to be constant within the triangular element; that is, it does not vary with positions within the element. Surprisingly, we found that the deformation gradient calculated based on this algorithm is identical to the one calculated based on the idea of linear transformation with an assumption of homogeneous strain field [28]. In fact, it can be shown that $\left[\begin{array}{ccc}x_{1}^{1}-x_{1}^{3} & x_{1}^{2}-x_{1}^{1} & x_{1}^{3}-x_{1}^{2} \\ x_{2}^{1}-x_{2}^{3} & x_{2}^{2}-x_{2}^{1} & x_{2}^{3}-x_{2}^{2}\end{array}\right]=\left[\begin{array}{ll}F_{11} & F_{12} \\ F_{21} & F_{22}\end{array}\right]\left[\begin{array}{ccc}c_{2} & c_{3} & c_{1} \\ -b_{2} & -b_{3} & -b_{1}\end{array}\right]$. Nevertheless, although the same results were achieved, the interpolation approach that we used in this study significantly reduced the computing time (1/10 of the linear transformation approach).

\section{References}

1. Ehrlich HP (1988) Wound closure - evidence of cooperation between fibroblasts and collagen matrix. Eye 2:149-157

2. Bell E, Ivarsson B, Merrill C (1979) Production of a tissue-like structure by contraction of collagen lattices by human-fibroblasts of different proliferative potential invitro. Proc Natl Acad Sci U S A 76(3):1274-1278

3. Harris AK, Stopak D, Wild P (1981) Fibroblast traction as a mechanism for collagen morphogenesis. Nature 290(5803): 249-251

4. Feng Z, Tateishi Y, Nomura Y, Kitajima T, Nakamura T (2006) Construction of fibroblast-collagen gels with orientated fibrils induced by static or dynamic stress: toward the fabrication of small tendon grafts. J Artif Organs 9(4):220-225

5. Grenier G, Remy-Zolghadri M, Larouche D, Gauvin R, Baker K, Bergeron F, Dupuis D, Langelier E, Rancourt D, Auger FA, Germain $\mathrm{L}$ (2005) Tissue reorganization in response to mechanical load increases functionality. Tissue Eng 11(1-2):90-100

6. Shi YL, Vesely I (2003) Fabrication of mitral valve chordae by directed collagen gel shrinkage. Tissue Eng 9(6):1233-1242

7. Thomopoulos S, Fomovsky GM, Holmes JW (2005) The development of structural and mechanical anisotropy in fibroblast populated collagen gels. J Biomech Eng-Transact Asme 127(5):742-750

8. Jhun CS, Evans MC, Barocas VH, Tranquillo RT (2009) Planar biaxial mechanical behavior of bioartificial tissues possessing prescribed fiber alignment. J Biomech EngTransact Asme 131 (8)

9. Lanir Y, Fung YC (1974) 2-Dimensional mechanicalproperties of rabbit skin.1. Experimental system. J Biomech 7(1):29-34

10. Bell BJ, Nauman E, Voytik-Harbin SL (2012) Multiscale strain analysis of tissue equivalents using a custom-designed biaxial testing device. Biophys J 102(6):1303-1312

11. Sun W, Sacks MS, Scott MJ (2005) Effects of boundary conditions on the estimation of the planar biaxial mechanical properties of soft tissues. J Biomech Eng 127(4):709-715

12. Waldman SD, Lee JM (2005) Effect of sample geometry on the apparent biaxial mechanical behaviour of planar connective tissues. Biomaterials 26(35):7504-7513

13. Hu JJ, Liu YC, Chen GW, Wang MX, Lee PY (2013) Development of fibroblast-seeded collagen gels under planar biaxial mechanical constraints: a biomechanical study. Biomech Model Mechanobiol. 12(5):849-868. doi:10.1007/s10237-012-0448-x

14. Mcculloch AD, Smaill BH, Hunter PJ (1987) Left-ventricular epicardial deformation in isolated arrested dog heart. Am J Physiol Heart Circ Physiol 252(1):H233-H241

15. Eilaghi A, Flanagan JG, Brodland GW, Ethier CR (2009) Strain uniformity in biaxial specimens is highly sensitive to attachment details. J Biomech Eng 131(9):091003. doi:10. $1115 / 1.3148467$

16. Knezevic V, Sim AJ, Borg TK, Holmes JW (2002) Isotonic biaxial loading of fibroblast-populated collagen gels: a versatile, low-cost system for the study of mechanobiology. Biomech Model Mechanobiol 1(1):59-67

17. Smits A, Van Hemelrijck D, Philippidis TP, Cardon A (2006) Design of a cruciform specimen for biaxial testing of fibre reinforced composite laminates. Compos Sci Technol 66(7-8):964-975. doi:10. 1016/j.compscitech.2005.08.011

18. Welsh JS, Adams DF (2002) An experimental investigation of the biaxial strength of IM6/3501-6 carbon/epoxy cross-ply laminates using cruciform specimens. Compos Part A-Appl S 33(6):829-839. doi:10.1016/S1359-835x(01)00142-7

19. Makris A, Vandenbergh T, Ramault C, Van Hemelrijck D, Lamkanfi E, Van Paepegem W (2010) Shape optimisation of a biaxially loaded cruciform specimen. Polym Test 29(2):216-223. doi:10.1016/j. polymertesting.2009.11.004

20. Holzapfel GA, Ogden RW (2009) On planar biaxial tests for anisotropic nonlinearly elastic solids. A continuum mechanical framework. Math Mech Solids 14(5):474-489

21. Pan B, Qian KM, Xie HM, Asundi A (2009) Two-dimensional digital image correlation for in-plane displacement and strain measurement: a review. Meas Sci Technol 20 (6)

22. Beda T (2005) Optimizing the Ogden strain energy expression of rubber materials. J Eng Mater-T Asme 127(3):351-353

23. Ogden RW, Saccomandi G, Sgura I (2004) Fitting hyperelastic models to experimental data. Comput Mech 34(6):484-502 
24. Lecompte D, Smits A, Sol H, Vantomme J, Van Hemelrijck D (2007) Mixed numerical-experimental technique for orthotropic parameter identification using blaxial tensile tests on cruciform specimens. Int J Solids Struct 44(5): $1643-1656$

25. Huang LX, Sun XS, Liu YH, Cen ZZ (2004) Parameter identification for two-dimensional orthotropic material bodies by the boundary element method. Eng Anal Bound Elem 28(2):109-121
26. Grediac M, Toussaint E, Pierron F (2002) Special virtual fields for the direct determination of material parameters with the virtual fields method. 2 - Application to in-plane properties. Int J Solids Struct 39(10):2707-2730

27. Humphrey JD, Vawter DL, Vito RP (1987) Quantification of strains in biaxially tested soft-tissues. J Biomech 20(1):59-65

28. Hu JJ, Fossum TW, Miller MW, Xu H, Liu JC, Humphrey JD (2007) Biomechanics of the porcine basilar artery in hypertension. Ann Biomed Eng 35(1):19-29 